\title{
Immune cell-derived opioids protect against neuropathic pain in mice
}

\author{
Dominika Labuz, Yvonne Schmidt, Anja Schreiter, Heike L. Rittner, \\ Shaaban A. Mousa, and Halina Machelska \\ Klinik für Anaesthesiologie und operative Intensivmedizin, Freie Universität Berlin, \\ Medizinische Fakultät Charité-Universitätsmedizin Berlin, Campus Benjamin Franklin, Berlin, Germany.
}

\begin{abstract}
The analgesic effects of leukocyte-derived opioids have been exclusively demonstrated for somatic inflammatory pain, for example, the pain associated with surgery and arthritis. Neuropathic pain results from injury to nerves, is often resistant to current treatments, and can seriously impair a patient's quality of life. Although it has been recognized that neuronal damage can involve inflammation, it is generally assumed that immune cells act predominately as generators of neuropathic pain. However, in this study we have demonstrated that leukocytes containing opioids are essential regulators of pain in a mouse model of neuropathy. About $30 \%-40 \%$ of immune cells that accumulated at injured nerves expressed opioid peptides such as $\beta$-endorphin, Met-enkephalin, and dynorphin A. Selective stimulation of these cells by local application of corticotropin-releasing factor led to opioid peptide-mediated activation of opioid receptors in damaged nerves. This ultimately abolished tactile allodynia, a highly debilitating heightened response to normally innocuous mechanical stimuli, which is symptomatic of neuropathy. Our findings suggest that selective targeting of opioid-containing immune cells promotes endogenous pain control and offers novel opportunities for management of painful neuropathies.
\end{abstract}

\section{Introduction}

Within inflamed tissues, a plethora of molecules such as protons, adenosine triphosphate, glutamate, neuropeptides (e.g., calcitonin gene-related peptide [CGRP], substance P), prostaglandins, bradykinin, cytokines, and chemokines can induce pain $(1,2)$. Concurrently, however, endogenous counterregulatory mechanisms are mounted. It has been established that somatic inflammatory (e.g., postoperative and arthritic) pain can be effectively controlled by the immune system, in both animals and humans $(3,4)$. This is mediated by extravasating leukocytes, which produce and liberate opioid peptides in inflamed tissues. The released opioids bind to opioid receptors on peripheral sensory neurons, resulting in the inhibition of noxious impulse propagation (5-17). Such effects are particularly interesting because they occur directly in peripheral tissues and, therefore, are free of side effects such as nausea, depression of breathing, cognitive impairment, dependence, and addiction mediated by opioid receptors in the CNS (3).

Neuropathic pain is a common consequence of nerve injuries caused by trauma such as amputation, entrapment, or compression. It is characterized by persistent burning or shooting sensations and heightened responses to normally noxious (hyperalgesia) and innocuous stimuli (allodynia). Despite increasing efforts, such pain remains poorly controlled, severely impacting patients' well-being (18-20), which makes new therapeutic approaches highly desirable. Research over the last decade has provided evidence on the association of traumatic peripheral nerve injuries with inflammatory reactions mobilizing the immune system (1,

Authorship note: Yvonne Schmidt and Anja Schreiter contributed equally to this work.

Conflict of interest: The authors have declared that no conflict of interest exists. Nonstandard abbreviations used: CCI, chronic constriction injury; CGRP, calcitonin gene-related peptide; CRF, corticotropin-releasing factor; CTOP, D-Phe-Cys-TyrD-Trp-Orn-Thr-Pen-Thr-NH ${ }_{2}$; ICI 174,864, $N, N$-diallyl-Tyr-Aib-Aib-Phe-Leu; NLXM, naloxone methiodide; norBNI, nor-binaltorphimine dihydrochloride.

Citation for this article: J. Clin. Invest. 119:278-286 (2009). doi:10.1172/JCI36246.
$2,21,22)$. Nerve damage can lead to recruitment of leukocytes and upregulation of inflammatory cytokines (e.g., TNF- $\alpha$, IL-1 $\beta$, IL-6) (1, 2, 21-25). Although a few reports addressed effects of antiinflammatory cytokines $(26,27)$, the majority of studies have concentrated on dampening of immune responses in neuropathy. Thus, deletion of IL- 6 , IL- $1 \alpha$, and IL- $1 \beta$ or chemokine receptor (CCR2) genes, anti-TNF treatments, and depletion of immune cells were reported to attenuate experimental neuropathic pain (1, $2,21,22,24,28-31)$. These studies clearly point to the conclusion that neuroimmune interactions are predominantly detrimental, leading to the generation of pain associated with nerve damage.

We now demonstrate in a mouse model of neuropathy that analgesic effects of immune cell-derived opioids are not restricted to somatic pain but are also critical for alleviation of pain resulting from injury of the nerves. Our results suggest that leukocytes using natural opioidergic painkillers serve as intrinsic regulators of neuropathic pain.

\section{Results}

Opioid-containing immune cells accumulate at the site of nerve injury. We used chronic constriction injury (CCI) of the sciatic nerve in mice, which resembles human neuropathy resulting from trauma of peripheral nerves, with some functional preservation of the innervation (e.g., nerve entrapment or compression) $(32,33)$. Mechanical allodynia can be especially distressing to patients, as tactile stimulation (for example, skin-to-clothes contact), and therefore pain, is inevitable. In our studies, application of von Frey filaments to the plantar surface of the hind paw innervated by the injured nerve resulted in profound mechanical allodynia that appeared on the first day and persisted up to 21 days following CCI. These symptoms occurred in the paw ipsilateral but not contralateral to CCI nor in sham-operated mice $(P<0.05$ and $P>0.05$, respectively; Supplemental Figure 1; supplemental material available online with this article; doi:10.1172/JCI36246DS1). Because it was previously reported that experimental neuropathic pain was attenuated by blocking of 
immune responses predominantly at the initiation of nerve injury $(1,24,29)$, we investigated the role of opioid-containing leukocytes at early (2-3 days) and later (14-15 days) stages of neuropathy.

For flow cytometric analysis of leukocytes, we prepared single-cell suspensions from sciatic nerves and hind paws. To exclude debris and nonhematopoietic cells, cell suspensions were stained with an $\mathrm{Ab}$ against CD45, a hematopoietic cell marker, and gated on $\mathrm{CD}_{4} 5^{+}$cells (Figure 1, A and B). Quantitative evaluation revealed significantly higher numbers of leukocytes (CD45 ${ }^{+}$cells) at injured and sham-operated nerves at 2 days compared with 14 days after surgeries $(P=0.021$ and $P<0.001$, respectively; Figure $1 \mathrm{~A})$. Importantly however, $\mathrm{CD} 45^{+}$ cell counts at the injured nerves were significantly higher compared with those at sham-operated nerves at both stages after surgeries $(P<0.001)$; there were practically no cells at sham-operated nerves at 14 days (Figure 1, A and B). Predominant immune cell infiltration of the nerve injury sites was further evident from the scarcity of leukocytes at nerves contralateral to CCI or sham surgery, at nerves from nonoperated animals, or in subcutaneous tissue of hind paws of nonoperated, CCI, and sham-operated mice (Figure 1A).

At the site of nerve injury, the total number of monocytes/macrophages was significantly higher compared with that of granulocytes at both 3 days $(P=0.005)$ and 15 days $(P<0.001)$ after CCI. T lymphocytes were absent at 3 days but were present at 15 days following CCI. Their total number was significantly lower compared with that of monocytes/macrophages $(P<0.001)$ and did not significantly differ from the number of granulocytes $(P=0.493)$ (Table $1)$. Thus, monocytes/macrophages dominated at both stages after CCI $(63.0 \% \pm 3.0 \%$ at 3 days and $60.0 \% \pm 1.5 \%$ at 15 days), granulocytes constituted $25.0 \% \pm 2.4 \%$ at 3 days and $11.0 \% \pm 1.2 \%$ at 15 days, while $\mathrm{T}$ lymphocytes were present only at a later phase of neuropathy $(9.0 \% \pm 0.8 \%)$, in agreement with previous reports $(23,24,27)$.

Next we searched for opioids in leukocytes accumulating at the operated nerves. To quantify them, we costained $\mathrm{CD} 45^{+}$cells with 3E7 Ab recognizing $\beta$-endorphin, Met-enkephalin, and dynorphin A. This analysis revealed that about $30 \%-40 \%$ of all $\mathrm{CD}^{4} 5^{+}$cells expressed opioid peptides, with the exception of sham-operated nerves at 14 days, when immune cells were essentially absent (Figure 1 , A and B). Minimal staining $(0.2 \%-1.4 \%)$ by an isotype-matched control Ab confirmed opioid specificity of the $3 \mathrm{E} 7 \mathrm{Ab}$ (Figure $1 \mathrm{~B}$ ). Opioid-containing leukocytes $\left(\mathrm{CD} 45^{+} 3 \mathrm{E} 7^{+}\right.$cells) accumulating at injured and sham-operated nerves were more abundant at 2 days than at 14 days $(P=0.012$ for injured nerves and $P<0.001$ for sham-operated nerves; Figure 1, A and B). Further, the numbers of $\mathrm{CD} 45^{+} 3 \mathrm{E}^{+}$cells at the injured nerves were significantly higher compared with those at sham-operated nerves at 2 days and 14 days after surgeries $(P<0.001$; Figure $1 \mathrm{~A})$. To differentiate between the 3 opioid peptides, we performed immunofluorescence using Abs selectively recognizing the respective opioids and found that cells accumulating at the ligated nerves expressed $\beta$-endorphin, Metenkephalin, and dynorphin A at both time points following CCI (Figure 1C). In addition, double immunofluorescence revealed that the majority of these cells coexpressed 2 peptides, i.e., $\beta$-endorphin with Met-enkephalin or dynorphin A or Met-enkephalin with dynorphin A at both stages of neuropathy (Supplemental Figure 2).

Opioid peptides expressed at the injured nerves alleviate neuropathic pain. In accordance with the previously shown release of opioid peptides from leukocytes by corticotropin-releasing factor (CRF) (13-15), the majority of $\beta$-endorphin-, Met-enkephalin-, and dynorphin Apositive cells coexpressed CRF receptors at both stages of nerve injury (Figure $1 \mathrm{C}$ ). We did not find CRF receptors in the sciatic nerve fibers (data not shown), similar to previous studies (34). Administration of CRF (5-20 ng at 2 days or 20-100 ng at 14 days) at the site of nerve injury dose-dependently and fully reversed mechanical allodynia measured at 30 minutes after CRF application, at early and later stages of neuropathy $(P<0.001$ and $P=0.003$, respectively; Figure 2A). The analgesic effects of the most effective doses of CRF ( $20 \mathrm{ng}$ at 2 days or $100 \mathrm{ng}$ at 14 days) peaked at 30 minutes and returned to the preinjection thresholds at 60 minutes ( 2 days after CCI) or 45 minutes (14 days after CCI) following CRF administration $(P<0.05$; Figure $2 \mathrm{~B})$. Furthermore, analgesia produced by the most effective CRF doses was dose-dependently reversed by coinjection with either CRF receptor antagonist ( $\alpha$-helical CRF; $0.125-2 \mathrm{ng} ; P<0.001$; Figure $2 \mathrm{C}$ ) or Abs against $\beta$-endorphin $(0.015-0.25 \mu \mathrm{g})$, Met-enkephalin $(0.0625-0.5 \mu \mathrm{g})$, or dynorphin A $(0.5-4 \mu \mathrm{g})(P<0.001)$ at both stages of neuropathy (Figure 2D). The thresholds to von Frey filaments in the paws contralateral to CCI were not significantly changed by any of these treatments $(P>0.05$; Figure 2A and data not shown). Also, the most effective CRF doses injected s.c. at the neck did not significantly change mechanical thresholds in paws ipsilateral $(P>0.05$; Figure $3 \mathrm{~A})$ or contralateral $(P>0.05$; data not shown) to the CCI, suggesting a lack of systemic CRF actions in our experiments. Although neuropathic pain could be attenuated by exogenous opiates injected into paws innervated by the injured nerves in previous studies (3), administration of the most effective CRF doses into the plantar surface of such painful paws did not produce analgesia in our study $(P>0.05$; Figure $3 \mathrm{~B})$. This argues against direct neuronal effects of CRF and is in line with the finding that CRF receptors were absent in peripheral nerves (see above) as well as with the scarcity of immune cells in these paws (Figure 1A). These results indicate that analgesic effects elicited by CRF injected at the site of nerve injury are mediated by local CRF receptors, $\beta$-endorphin, Met-enkephalin, and dynorphin $A$ and are associated with the presence of leukocytes.

Immune cells are the source of opioids that control neuropathic pain. To directly assess the functional significance of leukocytes, we investigated blockade of immune cell extravasation on CRF-mediated analgesia. ICAM-1 (CD54) is required for leukocyte migration (10, 35). Our immunostaining analysis revealed ICAM-1 expression in the blood vessels of the injured nerves and in the morphologically defined immune cells that accumulated at these nerves (Figure 4A). Daily i.p. treatment for 2 or 14 days (beginning the day of CCI) with a specific $\mathrm{mAb}$ against ICAM-1, but not with control IgG (both at $150 \mu \mathrm{g}$ per mouse), resulted in a significant drop in the number of all leukocytes $\left(\mathrm{CD} 45^{+}\right.$cells) and of opioid-containing immune cells $\left(\mathrm{CD} 45^{+} 3 \mathrm{E} 7^{+}\right)$at the site of nerve injury, at 2 days $(P<0.001$ for $\mathrm{CD} 45^{+}$and $\left.\mathrm{CD} 45^{+} 3 \mathrm{E} 7^{+}\right)$and at 14 days after $\mathrm{CCI}(P=0.036$ for $\mathrm{CD} 5^{+}$and $P=0.018$ for $\mathrm{CD} 45^{+} 3 \mathrm{E} 7^{+}$) (Figure 4B). The treatment with anti-ICAM-1 (12.5-150 $\mu \mathrm{g})$ did not significantly alter the nerve injury-induced mechanical allodynia (i.e., decreased von Frey thresholds after CCI and anti-ICAM- 1 but before CRF injection) $(P>0.05$; Figure $4 \mathrm{C})$. However, the analgesic effects of $\mathrm{CRF}$ injected at the site of nerve injury at 2 days ( $20 \mathrm{ng}$ ) or at 14 days (100 ng) after CCI were dose-dependently reversed by anti-ICAM-1 (12.5-150 $\mu \mathrm{g} ; P<0.001$; Figure 4D). Together, these findings point to the requirement for leukocytes to produce opioid-mediated analgesia in response to $\mathrm{CRF}$ applied at the site of nerve injury in neuropathic pain.

Peripheral opioid receptors mediate analgesia produced by immune cell-derived opioids in painful neuropatby. Effective opioid analgesia requires functional opioid receptors. Double immunofluorescence revealed colocalization of $\mu$-, $\delta$-, and $\kappa$-opioid receptors with CGRP 
A

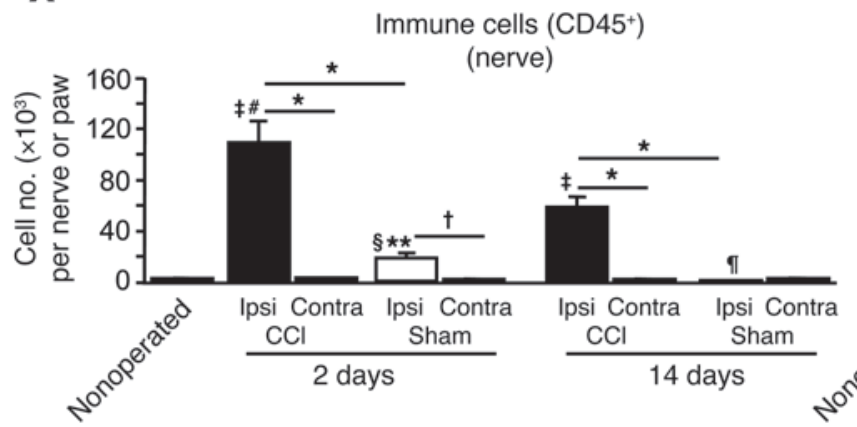

B
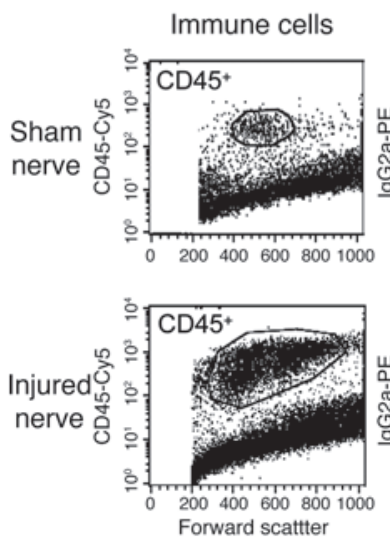

C
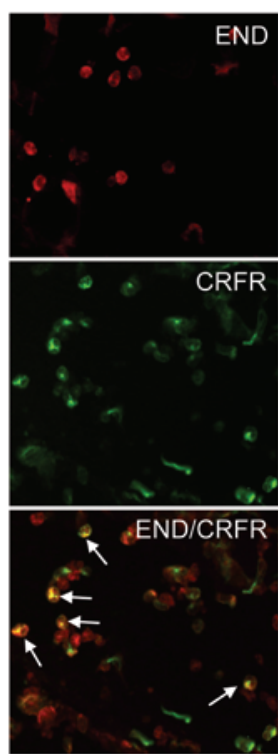

2 days

Opioid-containing immune cells
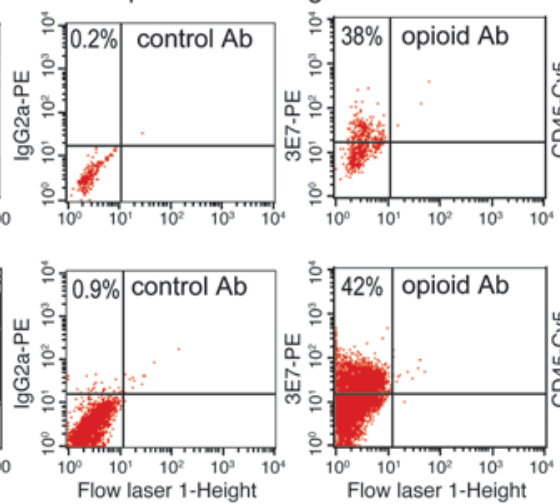

2 days
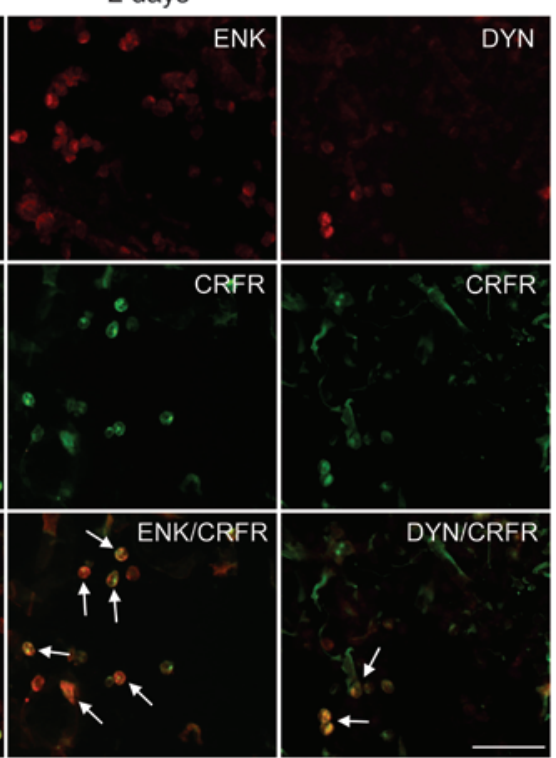

Immune cells (CD45+)

(ipsi paw)

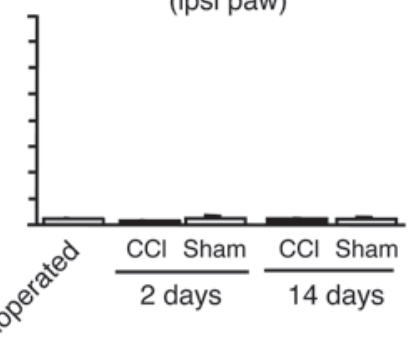

Opioid-containing immune cells $\left(\mathrm{CD} 45^{+} 3 \mathrm{E} 7^{+}\right)$

(ipsi nerve)

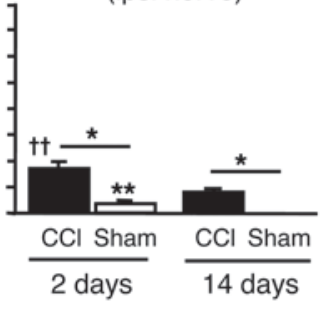

14 days

Opioid-containing immune cells
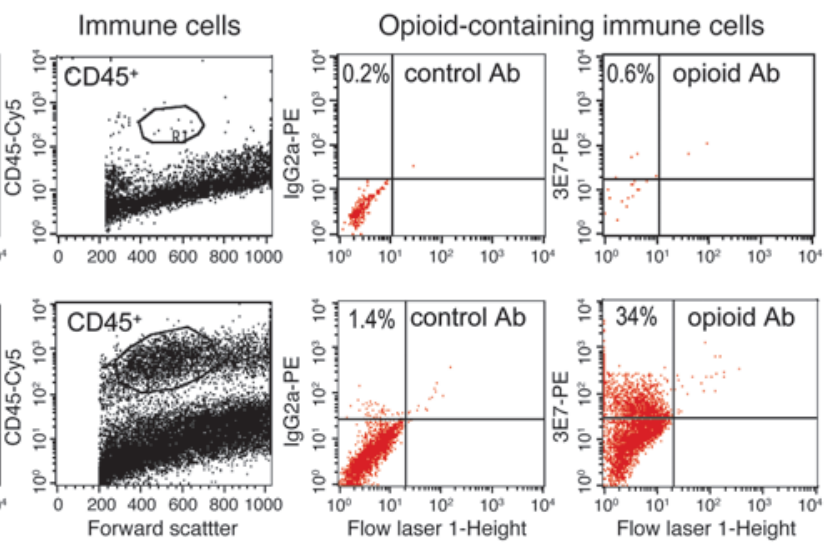

14 days
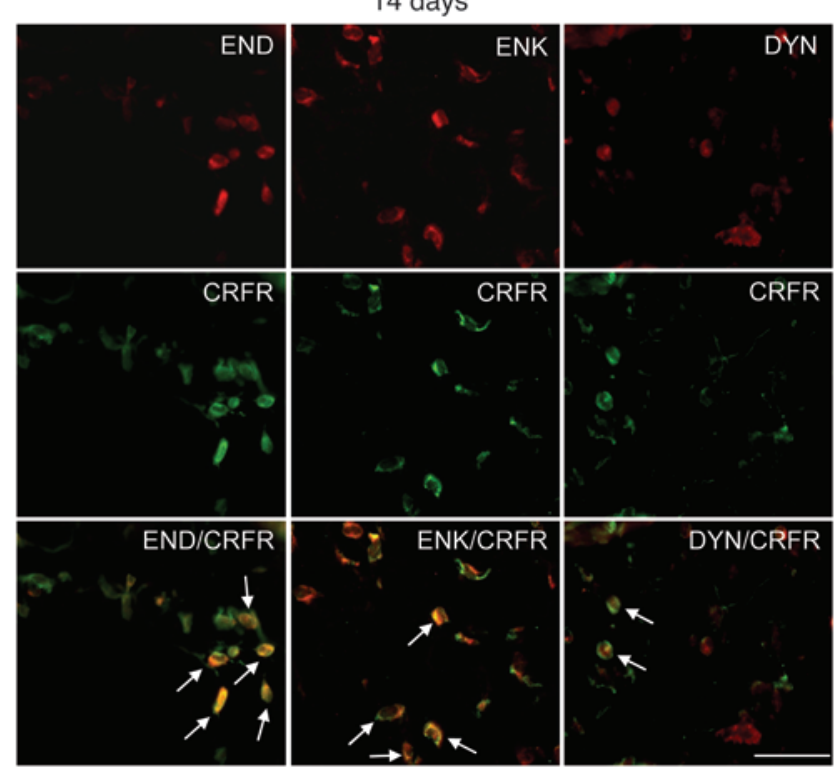

Figure 1

Expression of opioid peptides in immune cells accumulating at the nerve injury site. (A) Flow cytometric quantification of leukocytes (stained with $C D 45 \mathrm{Ab}$ ) and of $C D 45^{+}$cells expressing opioids (stained with $3 E 7 \mathrm{Ab}$ ) at nerves and in hind paws. ipsi and contra, nerves ipsilateral and contralateral to surgeries, respectively; Nonoperated, nerves or paws from nonoperated animals. ${ }^{\star} P<0.001, \dagger P=0.002 ; \ddagger P<0.001, \S P=0.002$, IP $=0.039$ versus nonoperated; ${ }^{*} P=0.021,{ }^{\star \star} P<0.001,{ }^{\dagger \dagger} P=0.012,2$ days versus 14 days $(t$ test). (B) Representative flow cytometric analysis of $\mathrm{CD} 45+$ cells (with CD45-phytoerythrin-cyanine dye 5 [Cy5] Ab) and of CD45+3E7+ cells (with 3E7-phytoerythrin Ab). Middle panels at 2 days and 14 days show control staining specificity of $3 E 7 \mathrm{Ab}$. (C) Representative double-immunofluorescence images showing coexpression of $\beta$-endorphin (END), Met-enkephalin (ENK), or dynorphin A (DYN) with CRF receptors (CRFR) in leukocytes (arrows) at injured nerves at 2 days and 14 days following $\mathrm{CCl}$. Scale bars: $50 \mu \mathrm{m}$. 
Table 1

Quantification of immune cell subpopulations at the injured nerves

\begin{tabular}{|c|c|c|c|c|}
\hline $\begin{array}{l}\text { Time after } \\
\text { nerve injury }\end{array}$ & $\begin{array}{l}\text { Immune cells } \\
\left(\mathrm{CD}_{4} 5^{+}\right)\left(\times 10^{3}\right)\end{array}$ & $\begin{array}{l}\text { Monocytes/macrophages } \\
\qquad\left(\times 10^{3}\right)\end{array}$ & $\begin{array}{l}\text { Granulocytes } \\
\qquad\left(\times 10^{3}\right)\end{array}$ & $\begin{array}{c}\text { T lymphocytes } \\
\left(\times 10^{3}\right)\end{array}$ \\
\hline $\begin{array}{l}3 \text { days } \\
15 \text { days }\end{array}$ & $\begin{array}{r}103 \pm 14 \mathrm{~A} \\
51 \pm 8.8\end{array}$ & $\begin{array}{l}64 \pm 10.3^{B, C, D} \\
30 \pm 5.2^{G}\end{array}$ & $\begin{array}{l}25 \pm 3.6^{\mathrm{E}, \mathrm{F}} \\
5.7 \pm 1.2\end{array}$ & $\begin{array}{c}0^{\mathrm{E}} \\
4.5 \pm 1.0\end{array}$ \\
\hline
\end{tabular}

Cell numbers were determined using flow cytometry. ${ }^{A} P=0.011$, $\mathrm{B} P=0.014$, compared with 15 days; $\mathrm{C} P=0.005$ compared with granulocytes at 3 days; $\mathrm{D} P<0.001$ compared with $\mathrm{T}$ lymphocytes at 3 days; $\mathrm{E} P<0.001$ compared with 15 days; $\mathrm{F} P<0.001$ compared with $\mathrm{T}$ lymphocytes at 3 days $(t$ test);

GP $<0.001$ compared with granulocytes and T lymphocytes at 15 days.

(a marker of sensory neurons) in the injured nerves at 2 and 14 days after CCI (Figure 5A). Further, blockade of $\mu$-, $\delta$-, and $\kappa$-opioid receptors at the site of injury by respective selective antagonists $\mathrm{D}$ Phe-Cys-Tyr-D-Trp-Orn-Thr-Pen-Thr-NH ${ }_{2}$ (CTOP; 0.015-0.25 $\mu \mathrm{g}$ ), $N, N$-diallyl-Tyr-Aib-Aib-Phe-Leu (ICI 174,864; 0.0625-2 $\mu \mathrm{g}$ ), and nor-binaltorphimine dihydrochloride (norBNI; 0.5-10 $\mu \mathrm{g}$ ) dosedependently reversed antiallodynic effects produced by CRF injected near injured nerves at both stages of neuropathic pain $(P=0.001$ for CTOP at 2 days and $P=0.002$ for CTOP at 14 days; $P=0.016$ for ICI 174,864 at 2 days and $P=0.004$ for ICI 174,864 at 14 days; $P<0.001$ for norBNI at both stages; Figure 5B). Moreover, the antiallodynic actions of CRF were dose-dependently blocked by coinjection of naloxone methiodide $(0.625-5 \mu \mathrm{g})$, an opioid receptor antagonist with limited access to the CNS $(36)(P<0.001$; Figure $5 \mathrm{~B})$, suggesting involvement of peripheral opioid receptors. Subcutaneous administration at the neck of CTOP $(0.25 \mu \mathrm{g})$, ICI $174,864(2 \mu \mathrm{g})$, norBNI $(10 \mu \mathrm{g})$, and naloxone methiodide (5 $\mu \mathrm{g})$ at doses that were the most effective after injections at the site of nerve injury (i.e., the most effective "near-nerve" doses) did not significantly change antiallodynic effects of CRF applied at the CCI site at 2 days $(P=0.978)$ and 14 days following nerve injury $(P=0.985$; Figure $5 \mathrm{~B})$. This confirms the observation that these antagonists act at opioid receptors expressed at the site of nerve injury. Von Frey filament thresholds in paws contralateral to the CCI were not significantly changed by any of the treatments $(P>0.05$; data not shown). Collectively, our results indicate that $\mu$-, $\delta$-, and
A
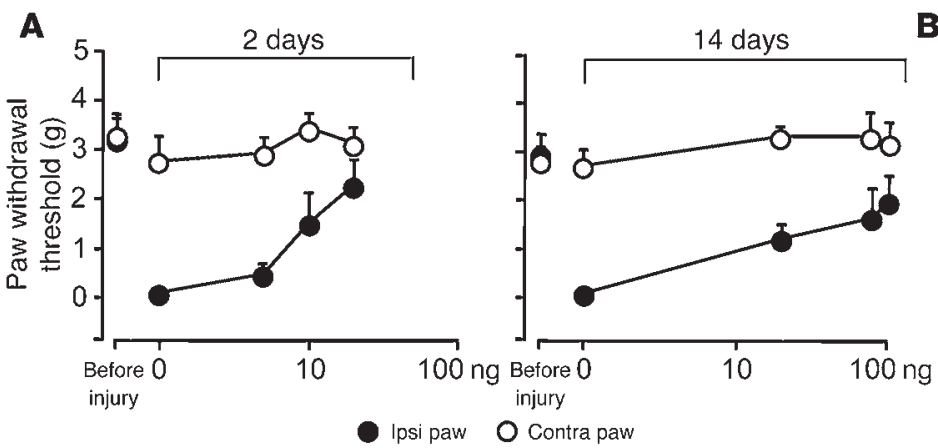

B

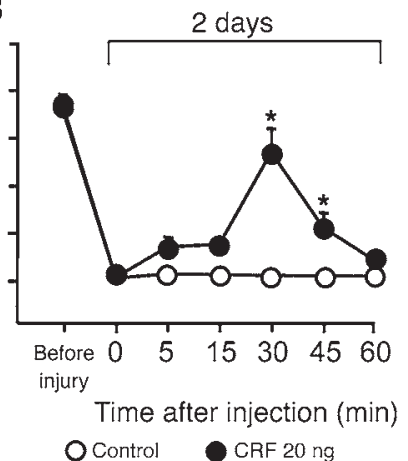

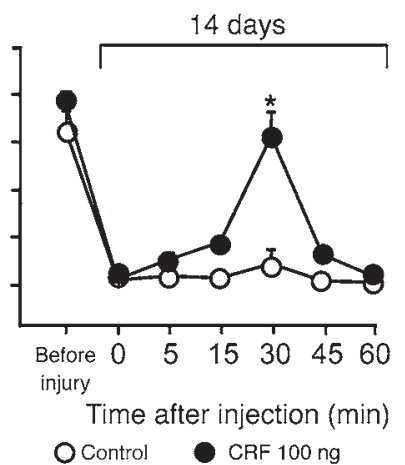

14 days

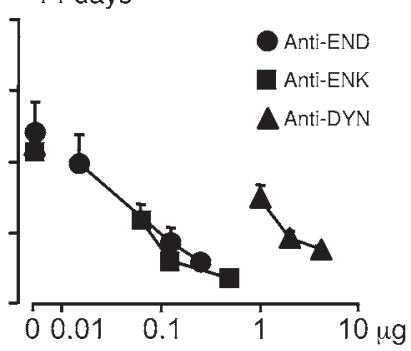

Figure 2

Analgesic effects produced by CRF injected at the site of nerve injury. (A) Dose-response relationships of CRF-induced analgesia measured 30 minutes after CRF application, in paws ipsilateral to $\mathrm{CCl}$ at 2 days $(5-20 \mathrm{ng})$ and at 14 days $(20-100 \mathrm{ng})$ following $\mathrm{CCl}(P<0.001$ and $P=0.003$, respectively; ANOVA, linear regression). No significant changes were observed in paws contralateral to $\mathrm{CCl}(P>0.05, \mathrm{ANOVA})$. (B) The time course of CRF-induced analgesia in paws ipsilateral to $\mathrm{CCl}$ at 2 days $(20 \mathrm{ng})$ and at 14 days (100 $\mathrm{ng})$ following CCl ( ${ }^{\star} P<0.05 \mathrm{compared}$ with 0 time point; repeated-measures ANOVA, Dunnett's test). There were no significant changes in control groups $(P>0.05$ compared with 0 time point; repeated-measures ANOVA). (C) Dose-dependent reversibility of CRF-induced (20 ng at 2 days or $100 \mathrm{ng}$ at 14 days) analgesia by coinjection of CRF receptor antagonist $\alpha$-helical CRF $(0.125-2 \mathrm{ng})$, in paws ipsilateral to $\mathrm{CCl}$ at 2 days and 14 days $(P<0.001$; ANOVA, linear regression). (D) Dose-dependent reversibility of CRF-induced (20 ng at 2 days or $100 \mathrm{ng}$ at 14 days) analgesia by coinjection of Abs against $\beta$-endorphin (Anti-END; 0.015-0.25 $\mu \mathrm{g}$ ), Met-enkephalin (Anti-ENK; 0.0625-0.5 $\mu \mathrm{g}$ ), or dynorphin A (Anti-DYN; 0.5-4 $\mu \mathrm{g}$ ), in paws ipsilateral to $\mathrm{CCl}$ at 2 days and 14 days after nerve injury $(P<0.001$; ANOVA, linear regression). 

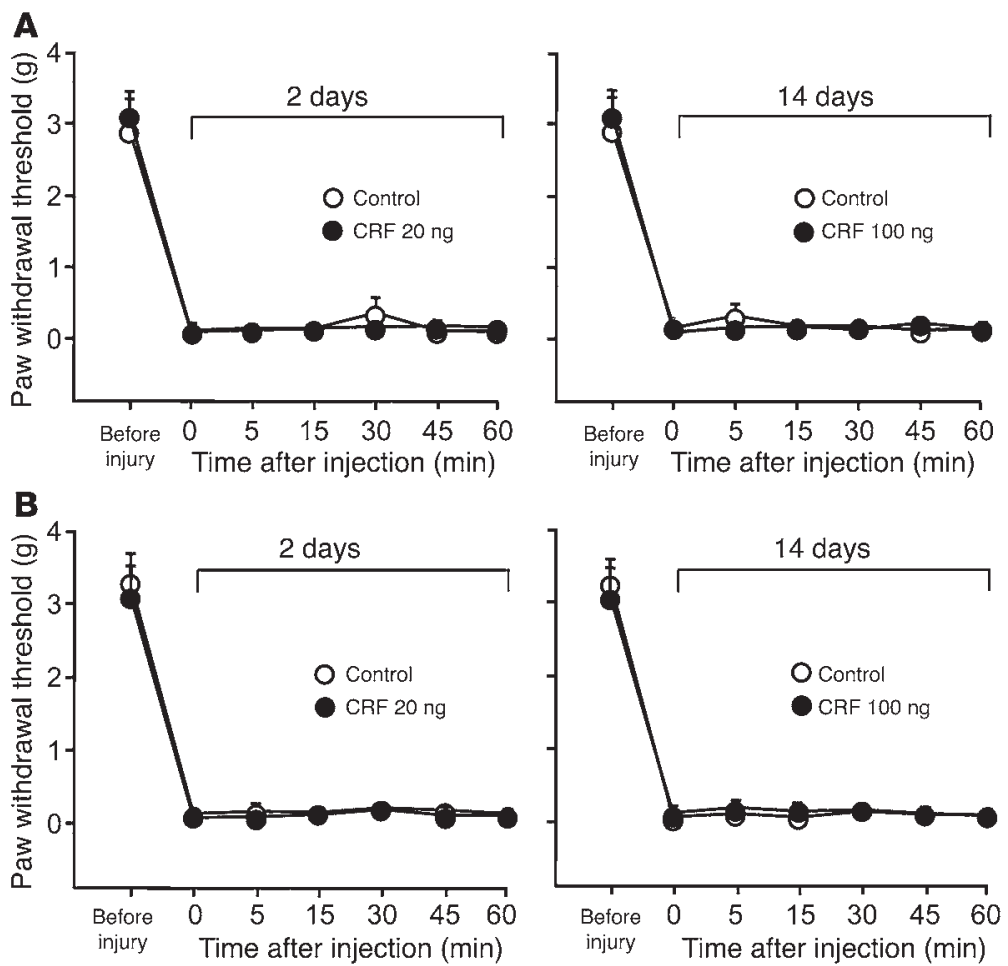

Figure 3

Lack of analgesic effects of CRF injected at sites distant to nerve injury. (A) Effects of CRF (20 ng at 2 days or $100 \mathrm{ng}$ at 14 days) injected s.c. at the neck on mechanical allodynia in paws ipsilateral to $\mathrm{CCl}$ as a function of time, at 2 days and 14 days after nerve injury. (B) Effects of CRF (20 ng at 2 days or $100 \mathrm{ng}$ at 14 days) injected into paws innervated by injured nerves on mechanical allodynia in these paws, as a function of time, at 2 days and 14 days after nerve injury. There were no significant changes after any treatments $(P>0.05$ compared with 0 time points; repeated-measures ANOVA).

$\kappa$-opioid receptors expressed in sensory fibers of the injured nerves directly at the site of CCI, are activated by opioid peptides derived from leukocytes to locally alleviate neuropathic pain.

\section{Discussion}

The need for neuropathic pain therapies is undisputed in view of the fact that it is poorly controlled and because of the well-known adverse effects (such as somnolence, dizziness, cognitive impairment, sleep and gait disturbances, and risk of cardiovascular complications) produced by currently used antidepressive and antiepileptic drugs $(19,20)$. In contrast to earlier assumptions, recent clinical trials have shown that patients with neuropathies may benefit from oral or intravenous opioids provided adequate individual dosage titration $(19,37)$. Nevertheless, such opioid use is limited by common side effects such as drowsiness, dizziness, sedation, nausea, and dependence mediated by opioid receptors in the CNS $(19,20,37)$. Conversely, selective activation of opioid receptors on peripheral nerves has a potential for effective and safe analgesia $(3,20,38)$. Similar approaches are also being applied using other drugs, such as cannabinoids (39), that otherwise are not safer or more efficacious than opioids $(19,37)$. Thus, the clinical efficacy of opioids as medications for neuropathic pain $(19,37)$ encourages the search to improve their analgesic profile. Our current study offers a possible advancement in neuropathic pain therapy based utilization of the physiological functions of leukocytederived opioids that represents control of painful neuropathies without CNS side effects.

We show here that opioid-containing immune cells provide effective suppression of mechanical allodynia, apparently by the secretion of opioids in response to CRF (13-15). This is supported by the findings that the 3 opioid peptides are coexpressed with CRF receptors in leukocytes and that CRF-induced analgesia can be reversed by blockade of CRF receptors along with neutralization of opioid peptides at injured nerves. Subsequently, the opioids activate $\mu$-, $\delta$-, and $\kappa$-opioid receptors expressed in nociceptors at the site of nerve damage, as demonstrated by the reversibility of CRF-induced analgesia by locally applied selective antagonists at each receptor type and by a peripherally acting opioid receptor antagonist. Interestingly, the CRF-mediated antiallodynic effects were reversed to a similar degree by each of the 3 Abs against opioid peptides or by each of the 3 opioid receptor antagonists. The information provided by the supplier (Bachem) and our own radioimmunoassay experiments testing whether any one of the Abs (e.g., against $\beta$-endorphin) recognized the other 2 (standard) peptides (e.g., Metenkephalin or dynorphin A) suggest that cross-reactivities of these Abs can be excluded. The similar effects of each of the Abs might be explained by the following scenario: (a) Before CRF injection, basal extracellular levels of opioid peptides maintain certain (low) nociceptive thresholds; (b) Following CRF application, levels of all 3 opioids are increased, resulting in blockade of allodynia; (c) Coinjection of CRF and an Ab against one of the peptides (e.g., $\beta$-endorphin) prevents this antiallodynic effect, although levels of the other 2 peptides (e.g., Met-enkephalin and dynorphin A) are still elevated. However, the total amount of available opioid molecules is lower than under condition $b$ and comparable to that under basal conditions (a), and therefore nociceptive thresholds return to the basal levels. Regarding the similar effects of each of the opioid receptor antagonists, interactions between opioid receptors subsequent to their potential oligomerization (40) may underlie the observation that antagonism at only one receptor type is sufficient to abolish the CRF-induced antiallodynic effects. More detailed studies are needed to clarify these issues in the future.

The absolute requirement for leukocytes to produce CRF-mediated analgesia at the site of nerve injury is demonstrated by the findings that (a) injection of CRF into the paws innervated by the ligated nerves did not produce analgesia, mirroring the scarcity of immune cells in this tissue; and (b) antiallodynic effects produced by CRF applied at the CCI site were abolished when the accumulation of opioid-containing leukocytes at the injured nerves was hindered by ICAM-1 blockade. The complete abolishment of CRF-mediated analgesia versus a partial (40\%-46\%) decrease in opioid-containing leukocyte numbers at the injured nerves in response to anti-ICAM-1 might be related to the fact that ICAM-1 participates not only in extravasation (35) but also in activation of immune cells (41). On the other hand, the finding that allodynia was not exacerbated by antiICAM-1 may be related to the strong CCI-induced allodynic effects already seen prior to anti-ICAM-1 treatment. Also, the differences between 2 days and 14 days regarding the total number of opioid- 
A

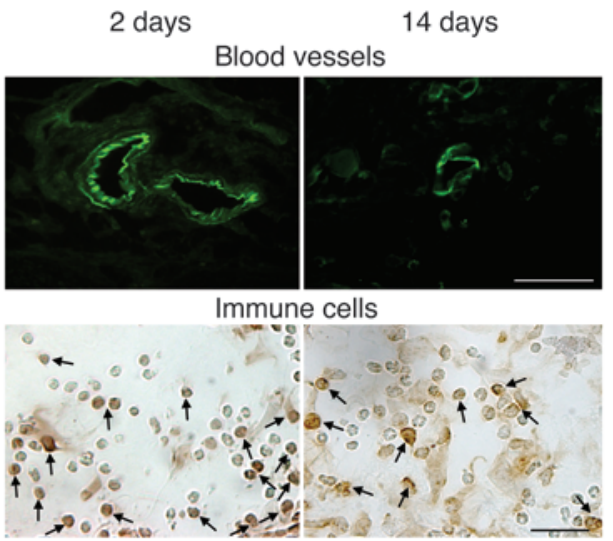

C

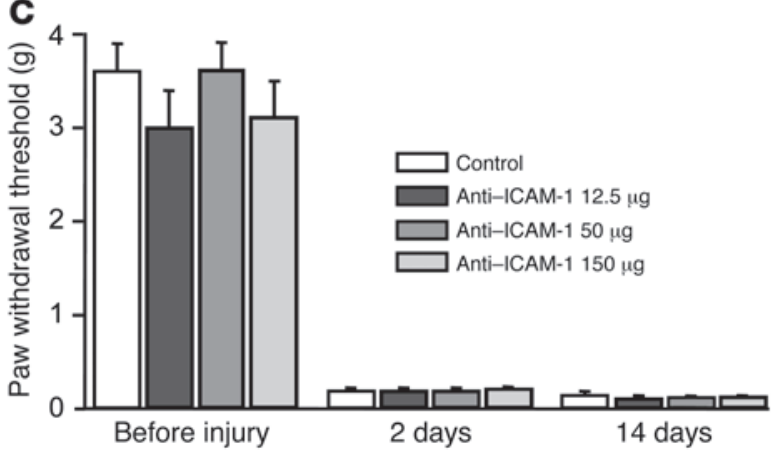

B

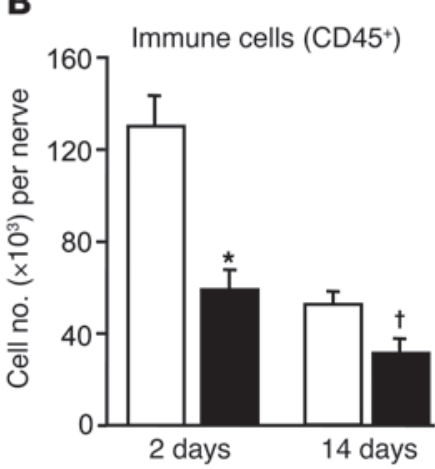

Opioid-containing



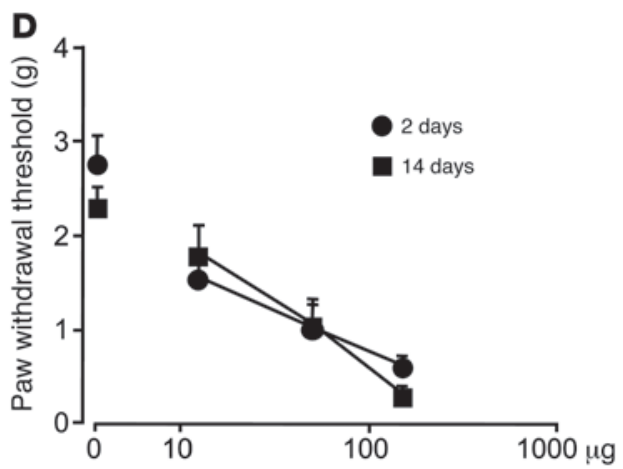

Figure 4

Contribution of opioid-containing immune cells to analgesia produced by CRF injected at the site of nerve injury. (A) Representative immunostaining images showing expression of ICAM-1 in blood vessels (in green) of injured nerves and in morphologically defined immune cells (arrows) at the injured nerves at 2 days and 14 days after $\mathrm{CCl}$. Scale bars: $50 \mu \mathrm{m}$. (B) Decrease in the numbers of all leukocytes (CD45+) and of opioid-containing leukocytes $\left(C D 45^{+} 3 E 7^{+}\right)$at the injured nerves by i.p. treatment with mAb against ICAM-1 (anti-ICAM-1; $150 \mu g$ per mouse). ${ }^{\star} P<0.001,{ }^{\dagger} P=0.036,{ }^{\ddagger} P=0.018$, control versus anti-ICAM-1 ( $t$ test). $(\mathbf{C})$ Lack of significant changes in mechanical allodynia in paws ipsilateral to $\mathrm{CCl}$ at 2 and 14 days following nerve injury by anti-ICAM-1 (12.5-150 $\mu$ g per mouse, i.p.; $P>0.05$, repeated-measures ANOVA). (D) Dose-dependent reversibility of CRF-induced (20 ng at 2 days or $100 \mathrm{ng}$ at 14 days) analgesia by anti-ICAM-1 (12.5-150 $\mu \mathrm{g}$ per mouse, i.p.) in paws ipsilateral to $\mathrm{CCl}(P<0.001$; ANOVA, linear regression).

containing leukocytes and the dosages of CRF suggest that higher CRF doses are required to produce analgesia by a lower number of opioid-containing immune cells. This is in line with experiments in somatic inflammatory pain $(10,16)$. Because CCI mice displayed allodynia before CRF application, it is reasonable to assume that nearnerve CRF doses reversing the allodynia are higher than the local extracellular concentration of endogenous CRF. To more precisely estimate these relationships, further studies, using, for example, local microdialysis, are required. The contribution of isoflurane anesthesia to antiallodynic effects of CRF is unlikely in our experiments, as solvent injections were ineffective and CRF blocked neuropathic pain only after applications at the CCI site but not after injections into the paw, although all types of administrations were performed under isoflurane anesthesia. Together, the results of this study show that immune cell-derived opioids are essential for the control of neuropathic pain. Clearly, the importance of the CRF-opioid system for neuropathic pain treatment awaits validation in patients. That the effects of CRF in our study were relatively short-lasting (30-45 minutes) does not necessarily reduce their clinical relevance, as already shown for exogenous and immune cell-mediated peripheral opioid analgesia in somatic inflammatory pain $(3,6,17)$.

Currently, immune cells are regarded primarily as generators of neuropathic pain $(1,2,21,22,24,29,33)$. Attenuation of neuro- pathic pain has been described in animals with reduced macrophage influx, genetic absence of $\mathrm{T}$ lymphocytes, depletion of neutrophils and macrophages, or in which mast cell degranulation is prevented $(24,27,29,33,42,43)$. This has been attributed to lower levels of inflammatory cytokines or chemokines activating sensory neurons $(1,2,21,22)$. Blockade of TNF production or of IL-1 receptors and deletion of either IL- 6 , IL- $1 \alpha$, and IL- $1 \beta$ or CCR 2 receptor genes were reported to decrease experimental neuropathic pain $(1,28,30$, 31 ). Nevertheless, other studies reported that macrophages and lymphocytes can stimulate tissue repair and motor function recovery or decrease hypersensitivity after peripheral nerve or spinal cord injury, mostly by removing tissue debris and producing neurotrophins or antiinflammatory cytokines $(27,44-46)$. Our results suggest that opioid peptides represent a novel component of positive effects of leukocytes in protection against nerve injury-induced pain. Interestingly, in line with these data are clinical observations that higher numbers of macrophages in nerve biopsy samples correlated with the absence of phantom pain after leg amputation, and low $\mathrm{CD}^{+} \mathrm{T}$ cell counts paralleled increased incidences of sensory neuropathies in patients infected with $\operatorname{HIV}(25,47)$. Clearly, immune cells can exert dual actions. Attenuation of neuropathic pain by leukocyte depletion in animal models most likely resulted from the prevention of early destructive actions of leukocytes, as this could be achieved 
A

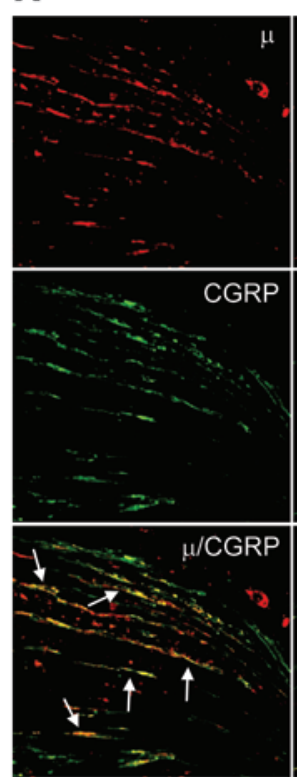

B

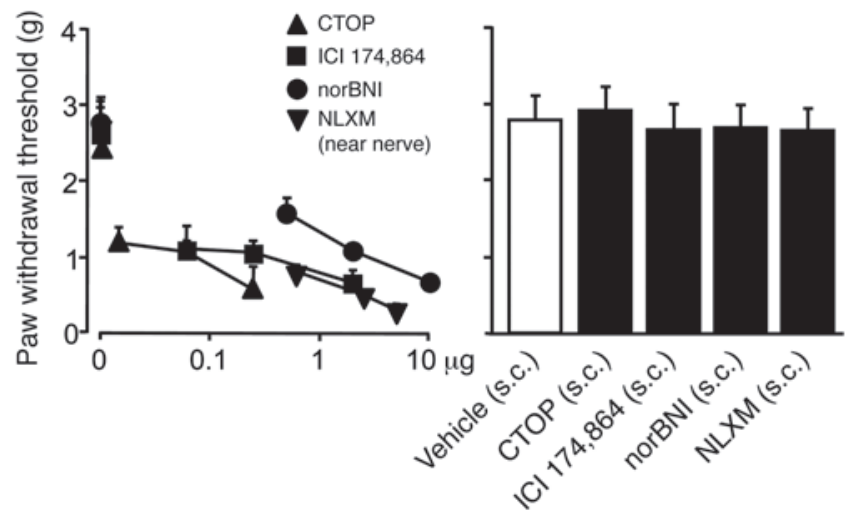

2 days
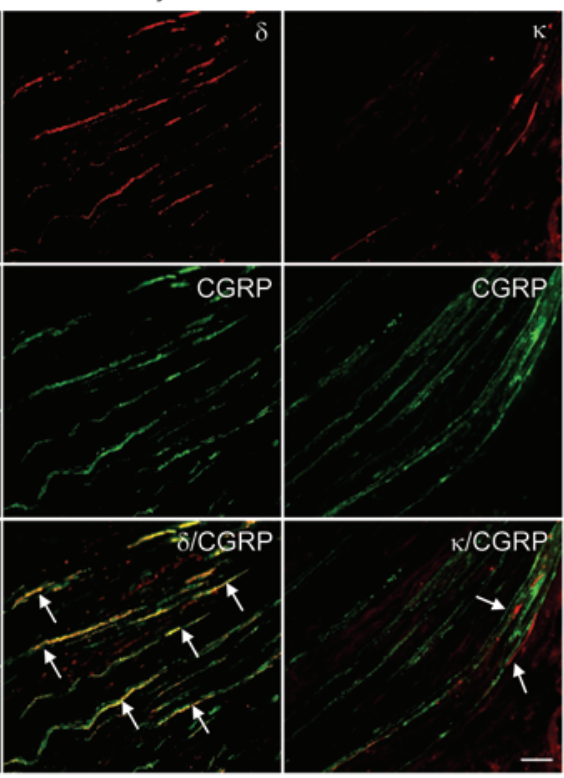

2 days

Figure 5

Contribution of opioid receptors expressed in injured nerves to analgesia produced by CRF injected at the site of injury. (A) Representative double-immunofluorescence images showing $\mu-, \delta$-, and $\kappa$-opioid receptor expression in sensory fibers (as determined by their coexpression with CGRP [arrows]) in the injured nerves at 2 and 14 days following CCI. Images are taken proximal to the most proximal ligature. Scale bars: $50 \mu \mathrm{m}$. (B) Left panels at 2 days and 14 days following CCl: Dose-dependent reversibility of CRF-induced (20 ng at 2 days or $100 \mathrm{ng}$ at 14 days) analgesia by coinjection of antagonists selective to $\mu$ - (CTOP; $0.015-0.25 \mu \mathrm{g}), \delta$ - (ICl 174,864; 0.0625-2 $\mu \mathrm{g}$ ), or $\kappa-$ opioid (norBNI; 0.5-10 $\mu \mathrm{g}$ ) receptors, as well as by an opioid receptor antagonist with limited access to the CNS, naloxone methiodide (NLXM; $0.625-5 \mu \mathrm{g})(P=0.001$ for CTOP at 2 days and $P=0.002$ for CTOP at 14 days; $P=0.016$ for $\mathrm{ICl} 174,864$ at 2 days and $P=0.004$ for $\mathrm{ICl} 174,864$ at 14 days; $P<0.001$ for norBNI and NLXM; ANOVA, linear regression). Right panels at 2 days and 14 days following CCI: Lack of reversibility of near-nerve CRF-induced (20 ng at 2 days or $100 \mathrm{ng}$ at 14 days) analgesia s.C. at the neck injected with the most effective near-nerve doses of CTOP $(0.25 \mu \mathrm{g}), \mathrm{ICI} 174,864$ $(2 \mu \mathrm{g})$, norBNI $(10 \mu \mathrm{g})$, and NLXM $(5 \mu \mathrm{g})(P>0.05$; ANOVA). The effects were measured in paws ipsilateral to $\mathrm{CCl}$.

only when cell depletion began at the initiation of neuropathy or when nerve injury was induced in animals already lacking immune cells. Simultaneously, however, the analgesic actions of leukocytederived opioids were probably hindered. This could be a reason for the rather moderate increases in pain thresholds after immunosuppression in most reports $(1,24,27,29,43)$ and for the lack of such effects in other studies (48). This could also explain our finding that mechanical allodynia was not alleviated when the number of immune cells was decreased in response to anti-ICAM-1 treatment. Hence, even though immune cell-derived inflammatory mediators might contribute to the initiation of neuropathic pain, they do not seem to play a major role in its maintenance. This brings into question the clinical relevance of antiinflammatory treatments in patients with established neuropathies.

In conclusion, the results of this study reveal what we believe to be a new target for specific exploitation of the beneficial effects of inflammation associated with nerve damage. Thus, selective activation of opioid-containing immune cells appears critical for the control of debilitating tactile sensitivity resulting from nerve injury. Furthermore, opioid cells can be protective not only during the initiation but also after establishment of painful neuropathy. Because peripheral excitatory mechanisms are essential for plas- 
ticity within the CNS (18), immune cell-derived opioids acting at peripheral opioid receptors in injured nerves might represent a first line of defense against painful inflammatory neuropathies. Finally, augmentation of such intrinsic peripheral inhibition might represent a novel mechanism and pharmacological approach for coping with neuropathic pain.

\section{Methods}

All animal experiments were approved by the local animal care committee (Landesamt für Gesundheit und Soziales Berlin, Germany).

Neuropathy and nociceptive testing. In deeply anesthetized (using isoflurane) male C57BL/6 mice (25-30 g) (bred at Charité-Universitätsmedizin Berlin, Campus Benjamin Franklin), the sciatic nerve was exposed at the level of the right mid-thigh, and 3 loose $4 / 0$ silk ligatures were placed around the nerve. The wound was closed with silk sutures. Sham operation was performed in a similar manner but without nerve ligation (33). Animals were habituated to the test cages daily starting 6 days prior to behavioral testing. Mechanical allodynia was evaluated with calibrated von Frey filaments (Stoelting) that were applied to the plantar surface of the hind paws using an up-down method (33). Testing began using a $3.9 \mathrm{mN}$ hair $(0.4 \mathrm{~g})$. If the animal withdrew the paw, the weaker hair was applied. In the case of no withdrawal, the next-stronger hair was applied. The maximum number of applications was 6-9, and the cut-off was $39.2 \mathrm{mN}(4 \mathrm{~g})$.

Flow cytometry. At 2-3 days and $14-15$ days after surgeries, mice $(n=6$ per group) were humanely killed with an overdose of isoflurane, and ligated parts of the sciatic nerves (approximately $1 \mathrm{~cm}$ long, including the ligation site and sites proximal and distal to it), corresponding sections of sham-operated nerves, sciatic nerves contralateral to CCI or sham surgery, and sciatic nerves from nonoperated animals, as well as subcutaneous tissue from hind paws innervated by sham-operated or injured nerves and tissue from hind paws of nonoperated mice, were collected. Single-cell suspensions were prepared $(7,10,16)$, and samples were stained with rat anti-mouse CD45 mAb conjugated with phytoerythrin-cyanine dye 5 (4 $\mu \mathrm{g} / \mathrm{ml}$; BD) to label all hematopoietic cells. To differentiate leukocyte subpopulations, we stained cell suspensions with specific phytoerythrinconjugated rat anti-mouse mAbs recognizing either granulocytes (Ly6) or T lymphocytes (CD3) (both at $4 \mu \mathrm{g} / \mathrm{ml}$; Bachem) and with FITC-conjugated $\mathrm{mAb}$ recognizing monocytes/macrophages (F4/80; $3 \mu \mathrm{g} / \mathrm{ml}$; Serotec). For intracellular stains, cells were prepared $(7,10,16)$ and incubated with $3 \mathrm{E} 7$ phytoerythrin-conjugated $\mathrm{mAb}$ recognizing the pan-opioid sequence Tyr-Gly-Gly-Phe at the N terminus of opioid peptides $(10 \mu \mathrm{g} / \mathrm{ml}$; Gramsch Laboratories). The specificity of the staining was verified by incubation of cell suspensions with appropriate isotype-matched control Abs. Absolute numbers of cells were calculated using Tru-COUNT tubes with known numbers of fluorescent beads. Data were acquired using a FACSCalibur and analyzed using CellQuest software (all from BD) $(7,10,16)$.

Immunostaining. At 2 and 14 days after CCI, mice $(n=3)$ were deeply anesthetized with isoflurane and perfused transcardially $(10,16,34)$. Ligated parts of the sciatic nerves (approximately $1 \mathrm{~cm}$ long, including the ligation site and sites proximal and distal to it) were dissected, postfixed, cryoprotected, embedded in OCT compound (Miles Inc.), frozen $(10,16,34)$, and cut into $10-\mu \mathrm{m}$-thick longitudinal sections that were then mounted on gelatin-coated slides. To examine the coexpression of opioid peptides with CRF receptors, we incubated the sections with goat anti-CRF receptor $\mathrm{Ab}$ recognizing both CRF receptor 1 and CRF receptor 2 (1:400; Santa Cruz Biotechnology Inc.) and with rabbit Abs against $\beta$-endorphin, Met-enkephalin (both at 1:500; Bachem), or dynorphin A (1:350; Abcam). To evaluate the coexpression of $\beta$-endorphin with Met-enkephalin or dynorphin A, we incubated the sections with guinea pig anti- $\beta$-endorphin $\mathrm{Ab}(1: 200)$ and rabbit anti-Met-enkephalin Ab (1:500) or rabbit anti-dynorphin A Ab (1:400) (all from Bachem), respectively. To analyze the coexpression of Met-enkephalin with dynorphin A, we incubated the sections with rabbit anti-Met-enkephalin Ab (1:500; Bachem) and goat anti-dynorphin A Ab (1:100; Santa Cruz Biotechnology Inc.). To assess expression of opioid receptors in sensory neurons, we incubated the sections with guinea pig anti-human (recognizing mouse) CGRP Ab (1:800; Bachem) and with rabbit Abs recognizing $\mu$-, $\delta$-, or $\kappa$-opioid receptors (all at 1:800; Abcam, Gramsch Laboratories, and Neuromics, respectively). All of these Abs were polyclonal. To evaluate ICAM-1 expression in the blood vessels, we incubated the sections with hamster anti-mouse ICAM-1 mAb (clone 3E2; 1:400; BD). The sections were then incubated with the respective secondary Abs: donkey anti-rabbit conjugated with Texas red, donkey anti-goat conjugated with FITC (1:200; Jackson ImmunoResearch Laboratories Inc.), goat anti-rabbit conjugated with Texas red, goat anti-guinea pig conjugated with FITC, or goat antihamster conjugated with FITC (1:200; Vector Laboratories). Thereafter, the sections were washed, mounted in Mowiol 4-88 (Carl Roth), and viewed under a fluorescence microscope (Zeiss) with appropriate filters.

To detect ICAM-1 in immune cells, we incubated the sections with antiICAM-1 (described above; 1:500). Staining was performed with a VECTASTAIN avidin-biotin peroxidase complex according to the manufacturer's instructions using goat anti-rabbit biotinylated secondary $\mathrm{Ab}$ and avidinbiotin peroxidase (VECTASTAIN Elite Kit; Vector Laboratories) $(16,34)$. For all experiments, specificity of the staining was confirmed by omission of the primary Abs or preabsorption (for 4 hours) of the primary Abs with the respective antigenic peptides (5- to 10-fold excess), which showed no staining (data not shown).

Behavioral experimental protocols. CRF (5-100 ng), $\alpha$-helical CRF $(0.125-2 \mathrm{ng})$,

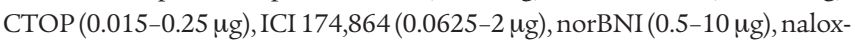
one methiodide (0.625-5 $\mu \mathrm{g}$ ) (all from Sigma-Aldrich); Abs against $\beta$-endorphin (0.015-0.25 $\mu \mathrm{g})$, Met-enkephalin (0.0625-0.5 $\mu \mathrm{g})$, and dynorphin A $(0.5-4 \mu \mathrm{g})$ (all from Bachem); anti-ICAM-1 Ab (clone 3E2; 12.5-150 $\mu$ g; $\mathrm{BD})$; and control rabbit IgG (12.5-150 $\mu \mathrm{g}$; Sigma-Aldrich) were dissolved in sterile water to obtain stock solutions, from which the required concentrations were prepared using $0.9 \% \mathrm{NaCl}$. Control groups were treated with either $0.9 \% \mathrm{NaCl}$ or control IgG in $0.9 \% \mathrm{NaCl}$. Substances were injected at the site of nerve injury $(30 \mu \mathrm{l})$ or intraplantarly into hind paws $(20 \mu \mathrm{l})$ under brief isoflurane anesthesia, s.c. at the neck $(0.1 \mathrm{ml} / 10 \mathrm{~g}$ body weight $)$ or i.p. (300 $\mu$ l per mouse) in awake animals. To decrease potential variability between near-nerve injections, we placed a polyethylene tube $2 \mathrm{~mm}$ from the tip around the $26 \mathrm{G}$ needle to ensure the same depth of needle insertion into the middle of the scar tissue after CCI surgeries. The site of these injections was verified by administration of $1 \%$ methylene blue $(30 \mu \mathrm{l})$, which reproducibly covered approximately $1 \mathrm{~cm}$ of the nerve, including the ligation site and sites proximal and distal to it. No animals were excluded from experiments in this study.

The development of mechanical allodynia was evaluated a day before and then daily at 1-7 days and at 14 and 21 days after CCI or sham surgery. The following experiments were performed at 2 and 14 days after CCI. CRF was injected either alone (at the site of nerve injury, intraplantarly or s.c.) or together (at the site of nerve injury) with either CRF receptor antagonist, opioid peptide Abs, or opioid receptor antagonists. To confirm a peripheral site of opioid receptor antagonist actions, the most effective near-nerve doses of CTOP $(0.25 \mu \mathrm{g})$, ICI 174,864 $(2 \mu \mathrm{g})$, norBNI $(10 \mu \mathrm{g})$, and naloxone methiodide $(5 \mu \mathrm{g})$ were applied s.c. immediately before CRF was applied at the CCI site. Ab against ICAM-1 was administered i.p. daily for 2 or 14 days (beginning the day of CCI). At 2 and 14 days after CCI and anti-ICAM-1 treatment, mechanical allodynia was measured, CRF was injected at the site of nerve injury, and mechanical allodynia was reevaluated 30 minutes later. All other dose-response relationships as well as effects of s.c. injected opioid receptor antagonists were assessed at 30 minutes after drug applications. 
The time course of CRF effects was measured before and at 5-60 minutes after injections. In all experiments, 6 animals per group were used.

Statistics. The data are expressed as mean \pm SEM. Two-sample comparisons were made with the 2 -tailed $t$ test. Dose-response relationships were analyzed with 1-way ANOVA followed by linear regression. Changes over time (more than 2 time points) were evaluated with repeated-measures ANOVA followed by Dunnett's test. Differences were considered significant if $P$ was less than 0.05 .

\section{Acknowledgments}

These studies were supported by a grant from the Deutsche Forschungsgemeinschaft (Klinische Forschergruppe 100/2) to H.
Machelska. We thank C. Stein, A. Kopf, and L. Carnduff for valuable comments on the manuscript.

Received for publication May 19, 2008, and accepted in revised form November 19, 2008.

Address correspondence to: Halina Machelska, Klinik für Anaesthesiologie und operative Intensivmedizin, Freie Universität Berlin, Medizinische Fakultät Charité-Universitätsmedizin Berlin, Campus Benjamin Franklin, Hindenburgdamm 30, D-12200 Berlin, Germany. Phone: 49-30-8445-3851; Fax: 49-30-8445-3826; E-mail: halina.machelska@charite.de.
1. Sommer, C., and Kress, M. 2004. Recent findings on how proinflammatory cytokines cause pain: peripheral mechanisms in inflammatory and neuropathic hyperalgesia. Neurosci. Lett. 361:184-187.

2. Marchand, F., Perretti, M., and McMahon, S.B. 2005 . Role of the immune system in chronic pain. Nat. Rev. Neurosci. 6:521-532.

3. Stein, C., Schäfer, M., and Machelska, H. 2003. Attacking pain at its source: new perspectives on opioids. Nat. Med. 9:1003-1008.

4. Machelska, H. 2007. Targeting of opioid-producing leukocytes for pain control. Neuropeptides. 41:285-293.

5. Stein, C., et al. 1990. Opioids from immunocytes interact with receptors on sensory nerves to inhibit nociception in inflammation. Proc. Natl. Acad. Sci. U. S. A. 87:5935-5939.

6. Stein, C., Hassan, A.H., Lehrberger, K., Giefing, J., and Yassouridis, A. 1993. Local analgesic effect of endogenous opioid peptides. Lancet. 342:321-324.

7. Rittner, H.L., et al. 2001. Opioid peptide-expressing leukocytes: identification, recruitment, and simultaneously increasing inhibition of inflammatory pain. Anesthesiology. 95:500-508.

8. Mousa, S.A., Shakibaei, M., Sitte, N., Schäfer, M., and Stein, C. 2004. Subcellular pathways of betaendorphin synthesis, processing, and release from immunocytes in inflammatory pain. Endocrinology. 145:1331-1341.

9. Machelska, H., Cabot, P.J., Mousa, S.A., Zhang, Q., and Stein, C. 1998. Pain control in inflammation governed by selectins. Nat. Med. 4:1425-1428.

10. Machelska, H., Mousa, S.A., Brack, A., Schopohl, J.K., Rittner, H.L., Schäfer, M., and Stein, C. 2002. Opioid control of inflammatory pain regulated by intercellular adhesion molecule-1. J. Neurosci. 22:5588-5596.

11. Machelska, H., et al. 2004. Selectins and integrins but not platelet-endothelial cell adhesion molecule-1 regulate opioid inhibition of inflammatory pain. Br. J. Pharmacol. 142:772-780.

12. Brack, A., et al. 2004. Control of inflammatory pain by chemokine-mediated recruitment of opioid-containing polymorphonuclear cells. Pain. 112:229-238.

13. Schäfer, M., Carter, L., and Stein, C. 1994. Interleukin 1 beta and corticotropin-releasing factor inhibit pain by releasing opioids from immune cells in inflamed tissue. Proc. Natl. Acad. Sci. U. S. A. 91:4219-4223.

14. Cabot, P.J., et al. 1997. Immune cell-derived beta-endorphin. Production, release, and control of inflammatory pain in rats. J. Clin. Invest. 100:142-148.

15. Cabot, P.J., Carter, L., Schäfer, M., and Stein, C. 2001. Methionine-enkephalin-and dynorphin Arelease from immune cells and control of inflammatory pain. Pain. 93:207-212.

16. Labuz, D., et al. 2006. Peripheral antinociceptive effects of exogenous and immune cell-derived endomorphins in prolonged inflammatory pain.
J. Neurosci. 26:4350-4358.

17. Likar, R., et al. 2007. Involvement of intra-articular corticotropin-releasing hormone in postoperative pain modulation. Clin. J. Pain. 23:136-142.

18. Scholz, J., and Woolf, C.J. 2002. Can we conquer pain? Nat. Neurosci. 5:1062-1067.

19. Dworkin, R.H., et al. 2007. Pharmacologic management of neuropathic pain: evidence-based recommendations. Pain. 132:237-251.

20. Stein, C., and Kopf, A. 2009. Anesthesia and treatment for chronic pain. In Miller's anesthesia. 7th edition. R.D. Miller, editor. Elsevier. Philadelphia, Pennsylvania, USA. In press

21. Watkins, L.R., and Maier, S.F. 2002. Beyond neurons: evidence that immune and glial cells contribute to pathological pain states. Physiol. Rev. 82:981-1011.

22. Moalem, G., and Tracey, D.L. 2006. Immune and inflammatory mechanisms in neuropathic pain. Brain Res. Rev. 51:240-264.

23. Cui, J.G., Holmin, S., Mathiesen, T., Meyerson, B.A., and Linderoth, B. 2000. Possible role of inflammatory mediators in tactile hypersensitivity in rat models of mononeuropathy. Pain. 88:239-248.

24. Perkins, N.M., and Tracey, D.J. 2000. Hyperalgesia due to nerve injury: role of neutrophils. Neuroscience. 101:745-757.

25. Stremmel, C., Horn, C., Eder, S., Dimmler, A., and Lang, W. 2005. The impact of immunological parameters on the development of phantom pain after major amputation. Eur. J. Vasc. Endovasc. Surg. 30:79-82.

26. Wagner, R., Janjingian, M., and Myers, R.R. 1998. Anti-inflammatory interleukin-10 therapy in CCI neuropathy decreases thermal hyperalgesia, macrophage recruitment, and endoneurial TNF-alpha expression. Pain. 74:35-42.

27. Moalem, G, Xu, K., and Yu, L. 2004. T lymphocytes play a role in neuropathic pain following peripheral nerve injury in rats. Neuroscience. 129:767-777.

28. Murphy, P.G., et al. 1999. Endogenous interleukin- 6 contributes to hypersensitivity to cutaneous stimuli and changes in neuropeptides associated with chronic nerve constriction in mice. Eur. J. Neurosci. 11:2243-2253.

29. Liu, T., van Rooijen, N., and Tracey, D.J. 2000. Depletion of macrophages reduces axonal degeneration and hyperalgesia following nerve injury. Pain. 86:25-32.

30. Abbadie, C., et al. 2003. Impaired neuropathic pain responses in mice lacking the chemokine receptor CCR2. Proc. Natl. Acad. Sci. U. S. A. 100:7947-7952.

31. Honore, P., et al. 2006. Interleukin-1alpha beta gene-deficient mice show reduced nociceptive sensitivity in models of inflammatory and neuropathic pain but not post-operative pain. Behav. Brain Res. 167:355-364

32. Bennett, G.J., and Xie, Y.K. 1988. A peripheral mononeuropathy in rat that produces disorders of pain sensation like those seen in man. Pain. 33:87-107.

33. Sommer, C., and Schäfers, M. 1998. Painful mono- neuropathy in C57BL/Wld mice with delayed Wallerian degeneration: differential effects of cytokine production and nerve regeneration on thermal and mechanical hypersensitivity. Brain Res. 784:154-162.

34. Mousa, S.A., Bopaiah, C.P., Richter, J.F., Yamdeu, R.S., and Schäfer, M. 2007. Inhibition of inflammatory pain by CRF at peripheral, spinal and supraspinal sites: involvement of areas coexpressing CRF receptors and opioid peptides. Neuropsychopharmacology. 32:2530-2542.

35. Von Andrian, U.H., and Mackay, C.R. 2000. T-cell function and migration. Two sides of the same coin. N. Engl. J. Med. 343:1020-1034.

36. Lewanowitsch, T., and Irvine, R.J. 2002. Naloxone methiodide reverses opioid-induced respiratory depression and analgesia without withdrawal. Eur. J. Pharmacol. 445:61-67.

37. Dellemijn, P. 1999. Are opioids effective in relieving neuropathic pain? Pain. 80:453-462.

38. Obara, I., et al. 2007. Local peripheral antinociceptive effects of 14-O-methyloxymorphone derivatives in inflammatory and neuropathic pain in the rat. Eur. J. Pharmacol. 558:60-67.

39. Agarwal, N., et al. 2007. Cannabinoids mediate analgesia largely via peripheral type 1 cannabinoid receptors in nociceptors. Nat. Neurosci. 10:870-879.

40. Gupta, A., Décaillot, F.M., and Devi, L.A. 2006. Targeting opioid receptor heterodimers: strategies for screening and drug development. AAPS J. 8:E153-E159.

41. Chirathaworn, C., et al. 2002. Stimulation through intercellular adhesion molecule- 1 provides a second signal for $\mathrm{T}$ cell activation. J. Immunol. 168:5530-5537.

42. Kleinschnitz, C., et al. 2006. T cell infiltration after chronic constriction injury of mouse sciatic nerve is associated with interleukin-17 expression. Exp. Neurol. 200:480-485.

43. Zuo, Y., Perkins, N.M., Tracey, D.J., and Geczy, C.L. 2003. Inflammation and hyperalgesia induced by nerve injury in the rat: a key role of mast cells. Pain. 105:467-479.

44. Perry, V.H., Brown, M.C., and Gordon, S. 1987. The macrophage response to central and peripheral nerve injury. A possible role for macrophages in regeneration. J. Exp. Med. 165:1218-1223.

45. Rapalino, O., et al. 1998. Implantation of stimulated homologous macrophages results in partial recovery of paraplegic rats. Nat. Med. 4:814-821.

46. Kerschensteiner, M., et al. 1999. Activated human T cells, B cells, and monocytes produce brain-derived neurotrophic factor in vitro and in inflammatory brain lesions: a neuroprotective role of inflammation? J. Exp. Med. 189:865-870.

47. Childs, E.A., et al. 1999. Plasma viral load and CD4 lymphocytes predict HIV-associated dementia and sensory neuropathy. Neurology. 52:607-613.

48. Rutkowski, M.D., et al. 2000. Limited role of macrophages in generation of nerve injury-induced mechanical allodynia. Physiol. Behav. 71:225-235. 